tion of the omitted point increases or diminishes the probability of guilt, according as $a+b$ is greater or less than $2 a$, or $a$ greater or less than $b$ : That is to say, the preponderance of the greater probability is diminished by the consideration of each unproved note. If, as was supposed to be generally the case in criminal trials, the probability from the evidence preponderates against the accused, then he does get a benefit from the due consideration of each unproved note, though very far from the benefit he would have gained by proving it in his favour.

\title{
2. On the Gradual Production of Luminous Impressions on the Eye, and other phenomena of Vision. By William Swan, F.R.S.E.
}

The object of this communication was to ascertain the relation between the apparent brightness of a light, and the time during which it acts on the eye. In order to examine the intensity of luminous impressions of short duration, the author made use of discs, having sectors of known angles cut out of their circumferences, which were made to revolve at known velocities between the eye and a luminous object. In this manner, the object is seen at each revolution of the disc for a short interval of time, of which the duration is easily ascertained. An instrument termed a selaometer (from $\sigma \varepsilon \lambda a \varsigma$, brightness), to indicate its use as a measure of the intensity of luminous impressions, was devised for the purpose of comparing the brightness of the flashes caused by the revolution of the disc, with a light of known intensity. This instrument consists of two screens, placed so as to face each other, having each a circular aperture of the same diameter, to which is fitted a piece of obscured glass. A disc, having a sector of a known angle, revolves in front of one of these screens, so that the aperture in it is visible at each revolution of the disc throughout the sector. The apertures are illuminated by gas flames behind them, which admit of having their distances from the screens varied, so as to increase or diminish the illumination of the apertures. A rectangular prism of glass is placed half way between the apertures, with its faces inclined at angles of $45^{\circ}$ to the line joining their centres ; so that they are seen in apparent contact by reflexion from the faces of the prism, and their relative brightness 
can thus be compared with great nicety. The light behind the rerolving disc is kept at a constant distance from the screen during an experiment; and, before causing the disc to revolve, the apertures are made equally bright by varying the distance of the other light from its screen. When the disc is put in motion, the apparent brightness of the aperture behind it is instantly diminished; and the equality of the apparent brightness of the apertures in the screens is restored, by increasing the distance of the light from the other screen. The ratio of the brightness of the impression produced by the light during the revolution of the disc, to the brightness of its impression, when seen by uninterrupted vision, is that of the squares of the distances of the other light from the aperture in its screen.

The following are the principal results obtained by means of this apparatus :-

(1.) When the eye receives, from a light of constant intensity, a succession of flashes of equal duration, which succeed each other so rapidly as to produce a uniform impression, this impression will also have a constant intensity, provided the number of flashes in a given time varies inversely with the duration of each flash.

(2.) The brightness of the impression produced by flashes of light of a given intensity, which succeed each other so rapidly as to produce a uniform impression on the eye, is proportional to the number of flashes in a given time.

(3.) When light of a given intensity acts on the eye for a short space of time, the brightness of the luminous impression on the retina is exactly proportional to the time during which the light continues to act. This law has been proved to be true for impressions lasting from $\frac{18}{8} \overline{4} \overline{2}$ to $\frac{1}{21}$ of a second. The intensity of the impression produced by light which acts on the eye for $\frac{1}{1} \overline{0}$ of a second, is almost exactly $\frac{1}{10}$ of the brightness of the light when seen by uninterrupted vision; and it is also ascertained that light requires about the tenth part of a second to produce its full effect on the eye.

(4.) It is found that lights of different intensity act on the eye with equal rapidity, so that even the light of the sun produces an impression with no greater rapidity than that of a common gas flame.

(5.) Rays of different refrangibility act on the eye with equal rapidity.

(6.) Since Professor Wheatstone's experiments have proved that 
the light of the electric spark of high tension continues for less than the millionth part of a second, and it has been shewn that the brightness of the impression, produced by light on the eye, increases in the exact arithmetical proportion of the time during which it continues to act on the retina, it follows that the apparent brightness of the electric spark is only $\bar{I} \overline{0} \bar{\sigma}^{1} \bar{o} \overline{0}$ of what it would become if the duration of the spark could be prolonged to $\frac{1}{10}$ th of a second. From the great apparent brilliancy of the nearly instantaneous electric spark of high tension, when compared with the sensibly continuous light of Voltaic electricity, it is inferred that the brightness of electrical light increases with the tension of the electricity.

\section{Note on the Refractive and Dispersive Powers of the Hu- mours of the Eye, determined by Experiment. By John Adie, Esq.}

The author's object in undertaking these experiments, was to discover if the achromatism of the eye could be accounted for by the differences in the dispersive ratios of the fluids forming that organ.

The indices for several of the fixed lines were determined in the aqueous humour; with the crystalline no satisfactory result could be obtained.

In subjecting the vitreous humour to experiment, only the strongest of the fixed lines could be seen, and that with great difficulty; one remarkable feature, however, was observed, viz., that on dividing the mass of humours two spectrums were formed, the one placed over the other, having a greater deviation, and, consequently, refractive power. Thus proving, that that humour is not of equal density throughout, as has heretofore been supposed.

The following Donations to the Library were announced:-

The London University Calendar for 1849. 8vo.-By the University.

The Ethnological Journal, No. 10. 8vo.-By the Editor.

Suite of the Collection of Hydrographic Charts, with Sailing Directions, \&c.-By the Lords Commissioners of the Admiralty. 